\title{
Solitary pulmonary caseating granulomas: A 5-year retrospective single-center analysis
}

\author{
HIDEHIRO WATANABE ${ }^{1}$, TOMONORI URUMA ${ }^{2}$, IKUO SEITA $^{2}$, TSUYOSHI OISHI ${ }^{2}$, \\ YUSUKE WATANABE $^{1}$, AYAKA TSUKIMORI ${ }^{1}$, YOSHITERU HAGA ${ }^{1}$, SHINJI FUKUSHIMA ${ }^{1}$, \\ AKIHIRO SATO $^{1}$, ITARU NAKAMURA ${ }^{1}$ and TETSUYA MATSUMOTO ${ }^{3}$ \\ ${ }^{1}$ Department of Infection Control and Prevention, Tokyo Medical University Hospital, Tokyo 160-0023; \\ ${ }^{2}$ Department of Infection Control and Prevention, Tokyo Medical University Ibaraki Medical Center, Inashiki, \\ Ibaraki 300-0395; ${ }^{3}$ Department of Microbiology, Tokyo Medical University Hospital, Tokyo 160-0023, Japan
}

Received October 4, 2016; Accepted January 10, 2017

DOI: $10.3892 / \mathrm{mco} .2017 .1244$

\begin{abstract}
Solitary pulmonary caseating granulomas (SPCGs) are a characteristic type of tuberculomas associated with infection with non-tuberculous mycobacteria (NTM) and other microbes; however, their significance remains unclear. The aim of the present study was to describe the clinical characteristics of patients with SPCGs in terms of diagnosis, presence of lung cancer and treatment status. A retrospective analysis of 17 immunocompetent patients with histopathologically confirmed caseating granulomas after undergoing video-assisted thoracoscopic surgery (VATS) was conducted at our center between 2011 and 2015. The patients comprised 10 men and 7 women with a mean age of $59.1 \pm 14.4$ years. Of the 17 patients, $14(82.4 \%)$ were asymptomatic and the lesions were discovered incidentally. In 2 patients the SPCGs were accompanied by a small satellite nodule (SPCG mean diameter, $16.2 \pm 5.1 \mathrm{~mm})$. Mycobacteria, including Mycobacterium tuberculosis (11.8\%), Mycobacterium avium (11.8\%) Mycobacterium kansasii (23.5\%) and other Mycobacterium spp. (5.9\%), were isolated from 9 of the patients (52.9\%). Concurrent lung cancer was present in 3 patients (17.6\%). When microbial agents could not be isolated, the interferon- $\gamma$ release assay was useful for diagnosis. Positron emission tomography was not found to be useful for differentiating SPCGs from lung cancer, or for differentiating tuberculomas from NTM pulmonary nodules (NTMPNs). NTMPNs in cases of SPCGs were diagnosed more frequently in men. The findings indicate that a course of observation may be sufficient for patients in whom an SPCG from NTM (NTMPN) is identified
\end{abstract}

Correspondence to: Dr Hidehiro Watanabe, Department of Infection Control and Prevention, Tokyo Medical University Hospital, 6-7-1 Nishishinjuku, Shinjuku-ku, Tokyo 160-0023, Japan E-mail: hw-nabe4@tokyo-med.ac.jp

Key words: tuberculoma, non-tuberculous mycobacterial pulmonary nodule, positron emission tomography, interferon- $\gamma$ release assay by VATS. However, the presence of concurrent lung cancer in certain cases indicates that malignancy should not necessarily be excluded, particularly in NTMPNs, and highlights the necessity of aggressive diagnosis by VATS.

\section{Introduction}

Solitary pulmonary caseating granulomas (SPCGs) are benign lesions that occur as mycobacterial pulmonary nodules (MPN) in the majority of cases (1); however, they have also been reported in some extremely rare cases of infection caused by fungi (2), Mycoplasma spp. (3), or Leishmania spp. (4). Among MPNs, tuberculomas caused by Mycobacterium tuberculosis (M. tuberculosis) account for $5-24 \%$ of all benign solitary pulmonary nodules (SPNs). Furthermore, $77-85 \%$ of all tuberculomas manifest as SPNs, with $\geq 2$ accompanying nodules or satellite lesions in $15-22 \%$ of the cases. These nodules most frequently occur in the upper lobes of the lungs. Clinically, $77 \%$ of the cases are asymptomatic and are discovered incidentally during health examinations $(5,6)$. Furthermore, it is extremely difficult to differentiate such nodules from lung cancer using computed tomography or the standardized uptake value (SUV) on positron emission tomography (PET) $(7,8)$. Currently, the only practicable method for the detection of PCGs is histopathological examination of specimens excised via video-assisted thoracoscopic surgery (VATS) or other surgical methods (9).

The post-VATS treatment of SPCG varies by case. Anti-tuberculosis drug treatment for $\sim 6$ months is currently the standard therapy for tuberculomas. However, this therapy is associated with a high frequency of drug-induced hepatotoxicity (DIH), with the subsequent requirement for hyposensitization therapy (10). Furthermore, a diagnosis of PCG by histopathological examination does not necessarily indicate the presence of a tuberculoma: It may also represent a non-tuberculous mycobacterial pulmonary nodule (NTMPN). The Mycobacterium avium (M. avium) complex accounts for the largest percentage of NTMPN cases, which reportedly occur more frequently in older age groups compared with tuberculomas $(11,12)$, although the precise age distribution is unknown. Furthermore, cases of PCG and concurrent lung 
cancer have also been reported $(6,13)$; therefore, a meticulous pathological examination of excised lesion tissue is required.

The aim of the present study was to perform a retrospective analysis of SPN cases histopathologically diagnosed as PCGs following VATS at the Tokyo Medical University Ibaraki Medical Center (Inashiki, Japan), in order to determine the clinical characteristics of patients with PCGs in terms of diagnosis (M.tuberculosis, NTM and others), presence of lung cancer and treatment status (clinical response and recurrence).

\section{Patients and methods}

Patients. The present retrospective study included a review and analysis of data from 17 patients aged $\geq 18$ years who presented with SPNs that were histopathologically diagnosed as PCGs following VATS at the Tokyo Medical University Ibaraki Medical Center between 2011 and 2015. All the patients were negative for human immunodeficiency virus on serological tests, and had no history of anti-tuberculosis drug treatment. The Tokyo Medical University Ibaraki Medical Center is a teaching hospital with $\sim 400$ beds, which is located in the southern part of Ibaraki Prefecture and serves a population of $\sim 400,000$. The study was conducted with the approval of the Tokyo Medical University Ibaraki Medical Center Ethics Committee (approval no. 15-33). Individual informed consent was not required given the retrospective nature of the study.

All 17 patients were assessed in terms of the following parameters: Age; gender; diagnostic modality; clinical manifestations; underlying disease; location, size and number of nodules; microbial isolates; treatment status; clinical course; SUV on PET; history of undergoing an interferon- $\gamma$ release assay (IGRA), namely the T-spot tuberculosis (TB) test; and histopathological analysis, including simultaneous occurrence of a cancer lesion.

Statistical considerations. All statistical analyses were performed with StatMate IV software (ATMS Co., Ltd., Tokyo, Japan). A descriptive analysis was performed for demographic and clinical characteristics, and results are presented as the mean \pm standard deviation (SD) for quantitative variables and numbers (percentages) for qualitative variables. For comparisons, the statistical significance of differences between tuberculoma, NTMPN and concurrent lung cancer groups was measured by one-way analysis of variance. All P-values were two-sided and $\mathrm{P}<0.05$ was considered to indicate statistically significant differences.

\section{Results}

Clinical background of the patients. The study population consisted of 10 men and 7 women, ranging in age from 29 to 79 years (mean, $59.1 \pm 14.4$ years) (Table I). The pulmonary nodules of 8 of the patients were discovered incidentally during a physical examination. In 6 patients, the nodules were discovered during follow-up for underlying diseases. No significant respiratory symptoms were observed upon diagnosis in 14 patients (82.4\%), whereas 3 patients (17.6\%) had overt symptoms (including bloody sputum in 1 patient, and cough and fever in 2 patients). Underlying diseases were present in 8 patients, including chronic renal failure $(n=2)$, diabetes mellitus $(n=2)$, hypertension $(n=1)$, chronic obstructive pulmonary disease $(n=2)$, sick sinus syndrome $(n=1)$, and postoperative lung cancer (under follow-up; $n=1$ ). Of the 17 patients, 6 had tuberculomas, including 1 male and 5 female patients, and the remaining 11 patients $(9$ men and 2 women, including the 3 patients with concurrent lung cancer) had NTMPNs.

Characteristics of SPNs. The main locations of the nodules were the right upper lobe $(n=6)$ and the left lower lobe $(n=4)$. In all patients with nodules in the left lower lobe, they were located in the S6 segment. The nodules were located in sites with a propensity for tuberculosis in 12 patients (70.6\%). The nodules were solitary in 15 patients, and 2 patients had SPCGs accompanied by a small satellite nodule. The occurrence of a small lesion was suspected upon re-examination in these 2 patients, whereas an infiltrative shadow was present in 1 patient, and 1 patient presented with a hilar nodule. The mean \pm SD diameter of the nodules was $16.2 \pm 5.1 \mathrm{~mm}$ (range, 8-27 mm). PET scans were performed in 7 patients, with the mean \pm SD SUV of the nodules measured as $7.9 \pm 3.7$ (range, 2.1-12.0). The mean SUV was $8.7 \pm 3.6$ in the 3 patients from

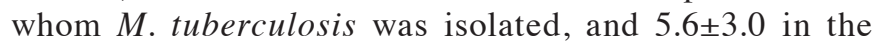
3 patients from whom NTMs were isolated (excluding the patients with concurrent lung cancer) (Table II).

Bacterial culture test and IGRA (T-spot TB test). Mycobacteria were isolated by culture in 9 patients $(52.9 \%)$, including M. tuberculosis in 2 patients (11.8\%), M. avium in 2 patients (11.8\%), Mycobacterium kansasii (M. kansasii) in 4 patients (23.5\%), and other NTM (unidentifiable as the 12 tested Mycobacterium spp.) in 1 patient (5.9\%). IGRA was performed in 11 patients, of whom 5 demonstrated positive test results, and 1 result was indeterminate. IGRA was positive in the 2 patients with culture-proven M.tuberculosis and among the 3 patients in whom no mycobacteria could be isolated. The result of the IGRA was indeterminate for 1 patient without mycobacteria, and negative for 1 patient with $M$. avium infection and for 2 patients with $M$. kansasii infection.

Histopathological findings and final diagnosis. In all 17 patients enrolled, the PCGs were diagnosed histopathologically. The final diagnosis was tuberculoma in 6 patients (35.3\%) and NTMPN in 11 patients (64.7\%) (Fig. 1). Lung cancer was also present in 3 patients (17.6\%); morphologically, the major lesion in all 3 cases was adenocarcinoma of the lung, and the mycobacterial nodules were located in the vicinity of the major malignant lesion (Fig. 2). NTMs were isolated from all 3 patients with lung cancer, including M. avium in 1 patient, M. kansasii in 1 patient, and an unknown species in 1 patient.

Treatment status. A total of 6 patients with tuberculoma received standard anti-tuberculosis drug therapy for 6 months. For 1 of these patients, the overall treatment period was extended to 12 months, as the patient developed DIH and required hyposensitization therapy, which required the anti-tuberculosis drugs to be increased gradually from a low initial dose. In all 3 patients with concurrent lung cancer, including 2 patients with T1aNOM0 stage Ia cancer and 1 with stage IV cancer, the nodules were non-tuberculous. The 2 patients with stage Ia 


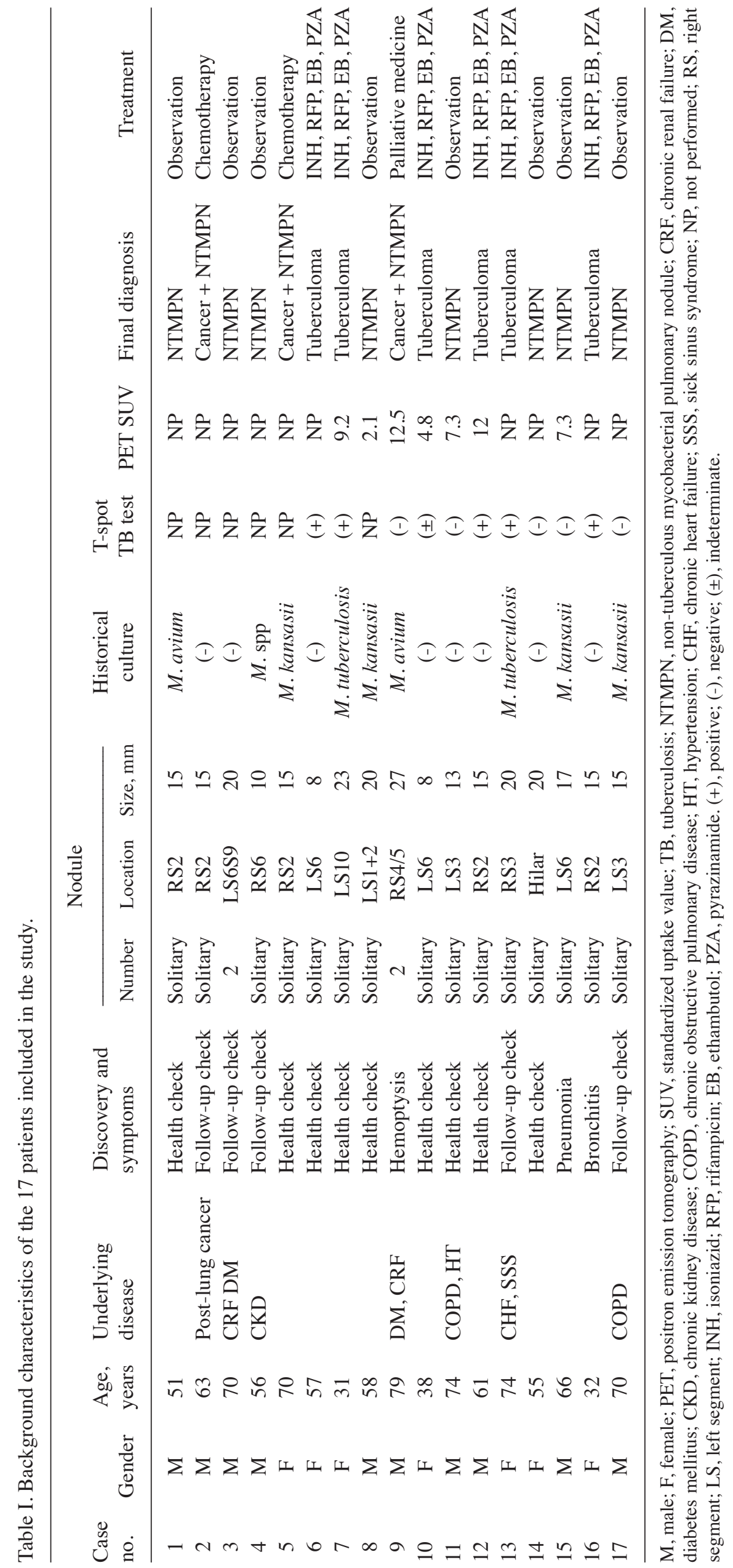



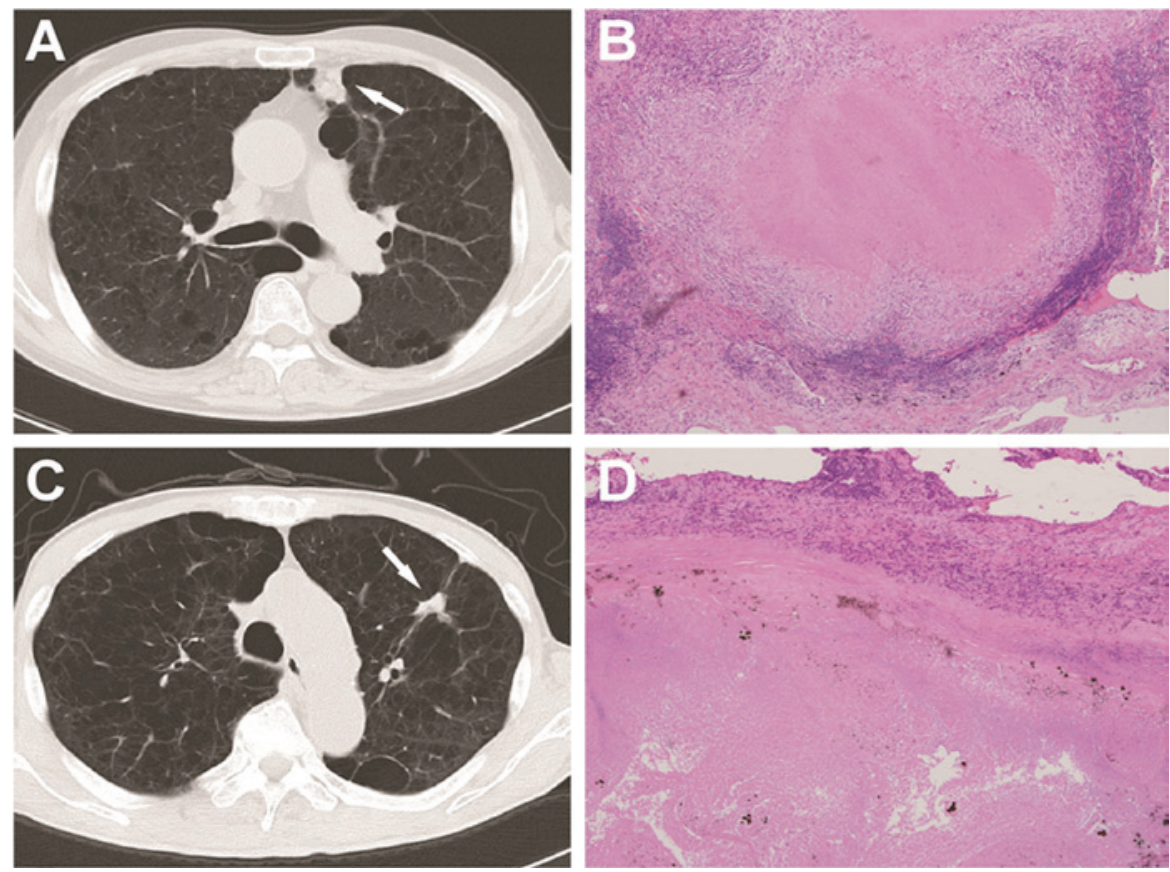

Figure 1. (A and B) A 74-year-old male patient (case no. 11) and (C and D) 70-year-old male patient (case no. 17) were diagnosed with a non-tuberculosis mycobacteria pulmonary nodule. Computed tomography imaging revealed a solitary nodule measuring 17 and $13 \mathrm{~mm}$ in diameter, respectively, in the left upper lung (A and C, arrows), and the video-assisted thoracoscopic surgery of the nodules revealed caseating granulomas (B and D; magnification, $\mathrm{x} 40$; hematoxylin and eosin stain).
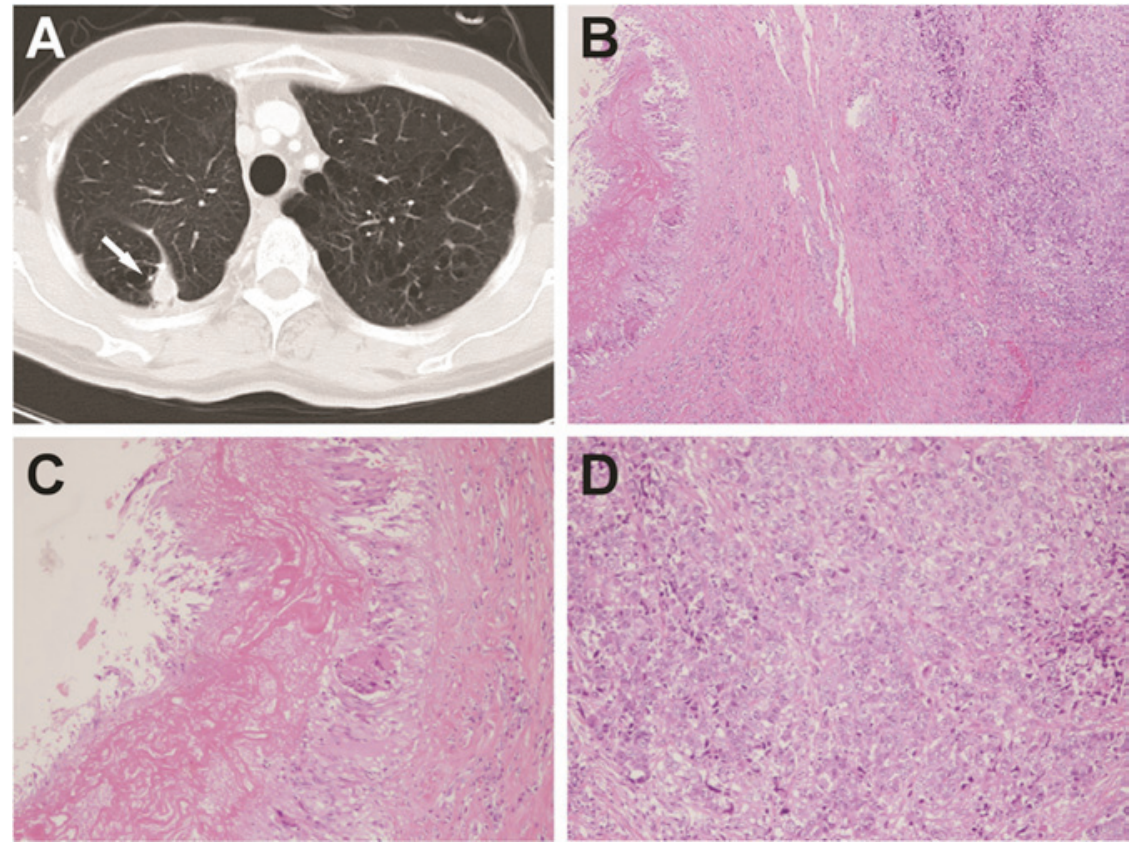

Figure 2. A 63-year-old male patient was diagnosed with a non-tuberculosis mycobacteria pulmonary nodule with concurrent lung cancer (case no. 2). (A) Computed tomography revealed a solitary nodule measuring $15 \mathrm{~mm}$ in diameter in the right upper lung (arrow). A caseating granuloma was observed surounding an adenocarcinoma in the nodule extracted by the video-assisted thoracoscopic surgery and subjected to hematoxylin and eosin staining; magnification, (B) x40 and (C and D) x100. (C) The periphery of the nodule consisted of a caseating granuloma surrounding (D) the adenocarcinomatous center.

cancer were treated by complete resection and followed up, while the patient with stage IV disease (with distant metastasis) only received palliative care at his own request. Of the 14 patients (6 with tuberculoma and 8 with NTMPN) without concurrent lung cancer, the follow-up period after treatment was $>3$ years in 6 patients, 2 years in 4 patients, and 1 year in 4 patients. None of the patients exhibited any evidence of relapse during follow-up.

\section{Discussion}

Tuberculomas reportedly account for $5-24 \%$ of all cases of SPN and are generally recognized as benign lesions (14). In an estimated $77.3 \%$ of the cases, tuberculomas are asymptomatic and are discovered incidentally during routine health examinations. It has been reported that tuberculomas manifest 
Table II. Comparison of cases by type.

\begin{tabular}{|c|c|c|c|c|}
\hline Variables & Tuberculoma & NTMPN & $\begin{array}{c}\text { Lung cance } \\
+ \\
\text { NTMPN }\end{array}$ & Total \\
\hline No. of cases & 6 & 8 & 3 & 17 \\
\hline Gender, no. male/female & $1 / 5$ & $7 / 1$ & $2 / 1$ & $10 / 7$ \\
\hline Age, years; mean \pm SD & $48.8 \pm 17.7$ & $62.5 \pm 8.51$ & $70.7 \pm 8.0^{\mathrm{a}}$ & $59.1 \pm 14.4$ \\
\hline Size, mm; mean \pm SD & $13.8 \pm 7.7$ & $16.3 \pm 3.7$ & $19.0 \pm 6.9$ & $16.1 \pm 5.4$ \\
\hline PET SUV; mean \pm SD (n) & $8.7 \pm 3.6(3)$ & $5.6 \pm 3.0(3)$ & $12.0(1)$ & $7.9 \pm 3.7(7)$ \\
\hline Isolation & 2 & 5 & 2 & 9 \\
\hline $\begin{array}{l}\text { No. positive T-spot } \mathrm{TB} / \text { tests } \\
\text { performed }\end{array}$ & $6 / 6$ & $0 / 4$ & $0 / 1$ & $6 / 11$ \\
\hline No. with/without underlying disease & $1 / 5$ & $4 / 4$ & $2 / 1$ & $7 / 10$ \\
\hline
\end{tabular}

${ }^{\mathrm{a}} \mathrm{P}<0.05$, tuberculoma vs. lung cancer + NTMPN patients. NTMPN, non-tuberculous mycobacterial pulmonary nodule; SD, standard deviation; PET, positron emission tomography; SUV, standardized uptake value.

as SPNs in $77-85 \%$ of the cases, and that $\geq 2$ nodules or satellite lesions are present in $15-22 \%$ of the cases. The nodules predominantly occur in the upper lobes of the lungs and have a mean diameter of $2-5 \mathrm{~cm}(5,6)$. It has also been reported that the likelihood of malignancy of an SPN increases with the increasing size of the nodule (15). In the present case series, the mean diameter of the nodules was $17.1 \pm 4.9 \mathrm{~mm}$; the estimated incidence of malignancy in nodules of this size range is $33-64 \%$ (15). However, lung cancer (including metastatic cancer) was suspected in all the present cases upon imaging diagnosis prior to VATS. PET has been reported to be useful for the diagnosis of malignancy in patients presenting with an SPN $(15,16)$. However, in the present cases, the mean SUV was $7.9 \pm 3.7$ in the patients that were examined by PET ( 7 of 17). Therefore, in all cases, the maximum SUV exceeded the reported SUV cutoff value of $\sim 2.5$ (17) for differentiating malignancy among nodular lesions. However, it is extremely difficult to differentiate between PCGs and lung cancer based on SUV alone $(10,16)$. In the 3 patients with NTMPNs and concurrent lung cancer, the mean SUV was 5.6 \pm 3.0 , which was not sufficient for the diagnostic differentiation of malignancy. No significant difference in the SUV was observed between

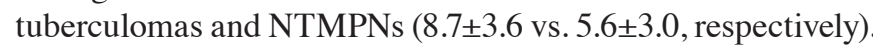
It may, therefore, be hypothesized that there is no difference in SUV between tuberculomas and NTMPNs, which both consist of local inflammatory reactions that produce nodular lesions. Generally, NTMs are more commonly encountered in postmenopausal women, and a number of theories have been postulated to explain this association, including low physical respiratory clearance (18), postmenopausal functional depression of macrophages (19), depressed villous movement (20) and a decrease in airway-epithelial ciliary movement caused by progesterone (21). According to previous reports, tuberculomas are more likely to occur in men compared with women $(5,6)$. Additionally, in the present study, patients with NTMPNs were older than those with tuberculomas, which is consistent with a previous report (11). Regarding gender, patients with NTMPNs comprised more men than women (Table II). These results are of considerable interest as they may suggest that the mechanism underlying nodular lesion formation differs from the mechanism of mycobacterial settlement in the case of NTMs. Further investigation, with a larger number of cases, is required.

In the present case series, the major lesion in all 3 patients (17.6\%) with concurrent lung cancer was pulmonary adenocarcinoma. Regarding the association between lung cancer and mycobacteria, various theories have been postulated, such as the post-mycobacteriosis-healing cicatrization theory and the theory of mycobacterial activation by cancer (22). More recently, latent tuberculosis has been associated with the risk of developing lung cancer (23). In the present study, SPCGs were detected in the vicinity of the lung cancer in all 3 cases. The mean age of the patients with concurrent lung cancer was $70.7 \pm 8.0$ years, which was significantly higher compared with that of the patients diagnosed with tuberculoma $(48.8 \pm 17.7$ years $)(\mathrm{P}<0.05)$. Our findings are highly suggestive of a potential association between lung cancer and latent mycobacterial infection. However, it is also possible that a breakdown of the local immune balance around the cancer lesion facilitated the emergence of the mycobacterial infection.

Mycobacterial species were identified in only 9 of the 17 patients $(52.9 \%)$ by detection/isolation from the excised tissues. In the remaining cases, no mycobacterial species were identified. The T-spot TB test, which is widely used for the diagnosis of tuberculosis, was employed in addition to the bacteriological diagnosis. The IGRA test is comparable with the QuantiFERON TB test regarding assay sensitivity and specificity, and it is also procedurally simpler $(24,25)$. The T-spot TB test was performed in 11 of the 17 patients in the present study. Of the 6 patients undergoing this test in whom mycobacteria could not be identified, 2 exhibited negative test results, while the test was positive in the remaining 4 patients, who were then finally diagnosed with tuberculoma. It has been reported that the T-spot TB test may also yield positive results in patients with infections caused by other mycobacteria, including M. kansasii, M. szulgai, M. marinum and M. gordonae (26). In the present study, however, the T-spot 
TB test was negative in all patients from whom NTM species, including M. kansasii, were isolated. Although it remains unclear why the T-spot TB test was negative in patients with $M$. kansasii infection, this test was considered useful for differentiating between tuberculoma and NTMPNs in patients with SPCGs identified by histopathology when no mycobacteria could be detected or isolated. Based on the present findings, it was concluded that no etiological factors for SPCGs other than mycobacteria were present in the current case series.

Standard anti-tuberculosis therapy is administered for the treatment of tuberculomas $(5,6,27)$. All 6 patients diagnosed with tuberculoma in the present study received anti-tuberculosis therapy. It has been reported that an estimated $15 \%$ of patients with tuberculoma who receive anti-tuberculosis treatment develop DIH (9). In the present study, 1 of the patients who received anti-tuberculosis treatment developed DIH, and hyposensitization therapy was undertaken, which proved efficacious. Regarding the management of NTMPNs, a previous study documented the case of a patient with an SPN caused by M. kansasii, who was followed up without anti-tuberculosis therapy following VATS and remained free from recurrence (28). None of the 8 patients with NTMPNs in the present study (excluding the 3 patients with NTMPNs associated with concurrent lung cancer) received anti-tuberculosis therapy, and no relapse was detected in any of these patients during the course of follow-up for $>1$ year. These results indicate that careful periodic follow-up may be sufficient for the management of NTMPNs, provided that the lesions are solitary.

The limitations of this study include a disparity in the process with which VATS was performed, the single-center design of the study and the limited number of cases, which did not allow for adequate assessment of SPCG. Accordingly, accumulation of data from multiple institutions, using pathological and microbiological analysis from VATS, is required for the study of SPCG.

In summary, a clinical evaluation was conducted for 17 patients with pulmonary nodules in whom SPCGs were histopathologically identified. Mycobacteria were identified following VATS in 9 of the patients (52.9\%). Finally, 6 patients were diagnosed with tuberculoma, 8 patients were diagnosed with NTMPNs and 3 patients were diagnosed with NTMPNs with concurrent lung cancer. Thus, NTMPNs accounted for $>60 \%$ of the patients with SPCGs, which also occurred more frequently in men. Lung cancer was suspected in all patients prior to VATS, and PET proved ineffective for diagnostic differentiation. Although, these results suggested that close periodic observation was sufficient for patients in whom SPCG from NTM (NTMPN) was identified by VATS, they also emphasize the necessity for aggressive VATS to obtain an accurate diagnosis. Furthermore, even if the central region of a solitary nodule is a malignancy, a caseous necrotic lesion may exist in the peripheral area. Therefore, it is necessary to examine the isolated tissue carefully.

\section{References}

1. Asakura T, Ishii M, Haraguchi M, Kamiyama I, Kohno M, Sakamaki H, Emoto K, Hayashi Y, Sugiura H, Kawada I, et al: Dry pleurisy complicating solitary pulmonary nodules caused by Mycobacterium avium: A case report. J Med Case Rep 9: 238, 2015.
2. Scott RS, Sutton DA and Jagirdar J: Lung infection due to opportunistic fungus, Phialemonium obovatum, in a bone marrow transplant recipient: An emerging infection with fungemia and Crohn disease-like involvement of the gastrointestinal tract. Ann Diagn Pathol 9: 227-230, 2005.

3. Linz D, Kessler S and Kane J: Pulmonary caseating granulomas associated with Mycoplasma pneumoniae pneumonia. South Med J 76: 1429-1432, 1983.

4. Aoun J, Habib R, Charaffeddine K, Taraif S, Loya A and Khalifeh I: Caseating granulomas in cutaneous leishmaniasis. PLoS Negl Trop Dis 8: e3255, 2014.

5. Lee HS, Oh JY, Lee JH, Yoo CG, Lee CT, Kim YW, Han SK, Shim YS and Yim JJ: Response of pulmonary tuberculomas to anti-tuberculous treatment. Eur Respir J 23: 452-455, 2004.

6. Hsu KY, Lee HC, Ou CC and Luh SP: Value of video-assisted thoracoscopic surgery in the diagnosis and treatment of pulmonary tuberculoma: 53 cases analysis and review of literature. J Zhejiang Univ Sci B 10: 375-379, 2009.

7. Chun EJ, Lee HJ, Kang WJ, Kim KG, Goo JM, Park CM and Lee $\mathrm{CH}$ : Differentiation between malignancy and inflammation in pulmonary ground-glass nodules: The feasibility of integrated (18)F-FDG PET/CT. Lung Cancer 65: 180-186, 2009.

8. Kawate E, Yamazaki M, Kohno T, Fujimori S and Takahashi H: Two cases with solitary pulmonary nodule due to non-tuberculous mycobacterial infection showing intense 18F-fluorodeoxyglucose uptake on positron emission tomography scan. Geriatr Gerontol Int 10: 251-254, 2010.

9. Chen S, Zhou J, Zhang J, Hu H, Luo X, Zhang Y and Chen H: Video-assisted thoracoscopic solitary pulmonary nodule resection after CT-guided hookwire localization: 43 cases report and literature review. Surg Endosc 25: 1723-1729, 2011.

10. Watanabe H, Uruma T, Seita I, Chikasawa Y, Kikuchi R, Itoh M, Aoshiba K, Nakamura H and Oishi T: Successful desensitization therapy involving fluoroquinolone for the treatment of a solitary tuberculoma: A case report and literature review. Mol Clin Oncol 5: 117-120, 2016.

11. Lim J, Lyu J, Choi CM, Oh YM, Lee SD, Kim WS, Kim DS, Lee $\mathrm{H}$ and Shim TS: Non-tuberculous mycobacterial diseases presenting as solitary pulmonary nodules. Int J Tuberc Lung Dis 14: 1635-1640, 2010.

12. Hahm CR, Park HY, Jeon K, Um SW, Suh GY, Chung MP, Kim H, Kwon OJ and Koh WJ: Solitary pulmonary nodules caused by Mycobacterium tuberculosis and Mycobacterium avium complex. Lung 188: 25-31, 2010.

13. Ashizawa K, Matsuyama N, Okimoto T, Hayashi H, Takahashi T, Oka T, Nagayasu T and Hayashi K: Coexistence of lung cancer and tuberculoma in the same lesion: Demonstration by high resolution and contrast-enhanced dynamic CT. Br J Radiol 77: 959-962, 2004.

14. Andreu J, Cáceres J, Pallisa E and Martinez-Rodriguez M: Radiological manifestations of pulmonary tuberculosis. Eur J Radiol 51: 139-149, 2004.

15. Zhan P, Xie H, Xu C, Hao K, Hou Z and Song Y: Management strategy of solitary pulmonary nodules. J Thorac Dis 5: 824-829, 2013.

16. Sathekge MM, Maes A, Pottel H, Stoltz A and van de Wiele C: Dual time-point FDG PET-CT for differentiating benign from malignant solitary pulmonary nodules in a TB endemic area. S Afr Med J 100: 598-601, 2010.

17. Opoka L, Kunikowska J, Podgajny Z, Szołkowska M, Błasińska-Przerwa K, Burakowska B, Oniszh K, Rudziński P, Bestry I and Roszkowski-Śliż K: Accuracy of FDG PET/CT in the evaluation of solitary pulmonary lesions-own experience. Pneumonol Alergol Pol 82: 198-205, 2014.

18. Chalermskulrat W, Gilbey JG and Donohue JF: Nontuberculous mycobacteria in women, young and old. Clin Chest Med 23: 675-686, 2002.

19. Miller L and Hunt JS: Sex steroid hormones and macrophage function. Life Sci 59: 1-14, 1996.

20. Comer MT, Leese HJ and Southgate J: Induction of a differentiated ciliated cell phenotype in primary cultures of Fallopian tube epithelium. Hum Reprod 13: 3114-3120, 1998.

21. Jain R, Ray JM, Pan JH and Brody SL: Sex hormone-dependent regulation of cilia beat frequency in airway epithelium. Am J Respir Cell Mol Biol 46: 446-453, 2012.

22. Kim YI, Goo JM, Kim HY, Song JW and Im JG: Coexisting bronchogenic carcinoma and pulmonary tuberculosis in the same lobe: Radiologic findings and clinical significance. Korean J Radiol 2: 138-144, 2001. 
23. Su VY, Yen YF, Pan SW, Chuang PH, Feng JY, Chou KT, Chen YM, Chen TJ and Su WJ: Latent tuberculosis infection and the risk of subsequent cancer. Medicine (Baltimore) 95: e2352, 2016.

24. Higuchi K, Sekiya Y, Igari H, Watanabe A and Harada N: Comparison of specificities between two interferon-gamma release assays in Japan. Int J Tuberc Lung Dis 16: 1190-1192, 2012.

25. Mancuso JD, Mazurek GH, Tribble D, Olsen C, Aronson NE, Geiter L, Goodwin D and Keep LW: Discordance among commercially available diagnostics for latent tuberculosis infection. Am J Respir Crit Care Med 185: 427-434, 2012.
26. Kuznetcova TI, Sauty A and Herbort CP: Uveitis with occult choroiditis due to Mycobacterium kansasii: Limitations of interferon-gamma release assay (IGRA) tests (case report and mini-review on ocular non-tuberculous mycobacteria and IGRA cross-reactivity). Int Ophthalmol 32: 499-506, 2012.

27. Laisaar T, Viiklepp P and Hollo V: Long-term follow-up after thoracoscopic resection of solitary pulmonary tuberculoma. Indian J Tuberc 61: 51-56, 2014

28. Abe M, Kobashi Y, Mouri K, Obase Y, Miyashita N, Nakata M and Oka M: Solitary pulmonary nodule due to Mycobacterium kansasii. Intern Med 50: 775-778, 2011. 